\title{
Discordance of serological and sonographic markers for Hashimoto's thyroiditis with gold standard histopathology
}

\author{
Haixia Guan ${ }^{1,2, *}$, Nathalie Silva de Morais ${ }^{2,3,4, *}$, Jessica Stuart ${ }^{2}$, Sara Ahmadi², \\ Ellen Marqusee ${ }^{2}$, Mathew I Kim² and Erik K. Alexander ${ }^{2}$
}

${ }^{1}$ Department of Endocrinology and Metabolism, The First Hospital of China Medical University, Shenyang, China, ${ }^{2}$ Thyroid Section, Brigham \& Women's Hospital \& Harvard Medical School, Boston, MA, USA, ${ }^{3}$ Endocrinology Service, Instituto Nacional de Câncer and Universidade Federal do Rio de Janeiro, Rio de Janeiro, Brazil, and ${ }^{4}$ Endocrinology Service, Instituto Estadual de Diabetes e Endocrinologia Luiz Capriglione, Rio de Janeiro, Brazil

*(H Guan and N S de Morais contributed equally to this work)
Correspondence should be addressed to N S de Morais or E Alexander Email nathalieaos@gmail.com or ekalexander@bwh. harvard.edu

\begin{abstract}
Objective: To investigate the concordance of serologic and sonographic evidence of Hashimoto's thyroiditis with its gold standard histopathologic identification.

Design: We performed a retrospective analysis on a cohort of 825 consecutive patients in whom TPOAb and thyroid ultrasound were performed, and in whom thyroid nodule evaluation led to surgical and histopathologic analysis. The presence or absence of Hashimoto's thyroiditis on histopathology was correlated with serologic and sonographic markers. We further assessed the impact of low versus high titers of TPOAb upon this concordance.

Results: Of 825 patients, 277 (33.5\%) had histologic confirmation of Hashimoto's thyroiditis, 235 patients (28.4\%) had elevated serum levels of TPOAb, and 197 (23.8\%) had sonographic evidence of diffuse heterogeneity. Of those with histopathologic evidence, only 64\% had elevated TPOAb (sensitivity: 63.9\%; specificity: 89.4\%), while only 49\% were sonographically diffusely heterogeneous (sensitivity: 49.1\%; specificity: $88.9 \%$ ). A subset of only 102 of 277 (37\%) with histologically proven Hashimoto's thyroiditis was positive for both TPOAb and diffusely heterogeneous. Concordance analysis demonstrated that TPOAb and histopathology had higher agreement $(\kappa=0.55)$ than did ultrasound and histopathology $(\kappa=0.40)$ for the diagnosis of Hashimoto's thyroiditis. Higher titers of TPOAb correlated with a higher likelihood of Hashimoto's thyroiditis, with a best cutoff of 2.11-fold the upper normal level of TPOAb.

Conclusion: Only moderate concordance exists between serological evidence of Hashimoto's thyroiditis and histopathologic findings, though it increases with higher TPOAb concentration. Diffuse heterogeneity on ultrasound is a less-sensitive diagnostic tool than elevated TPOAb.
\end{abstract}

\section{Introduction}

Hashimoto's thyroiditis, a chronic inflammatory disorder of the thyroid gland, was first described in Japan by Dr. Hakaru Hashimoto over a century ago (1). It was long considered a rare disease as the diagnosis was mostly based on hypothyroid symptoms or, less commonly, was identified following histopathological examination of thyroidectomy specimens. However, following the introduction of thyroid function and thyroid antibodies tests, its incidence rapidly increased. Hashimoto's disease is now considered the most common autoimmune disease and the most common endocrine disorder in welldeveloped countries $(2,3,4)$. 
In the clinical setting, the diagnosis of Hashimoto's thyroiditis is established by detecting a combination of clinical, serological, and sonographic features. Although histological findings of a diffuse lymphocytic infiltration with numerous lymphoid follicles and germinal centers remains the gold standard for the diagnosis of Hashimoto's thyroiditis, thyroidectomy is rarely performed in this setting and virtually never applied for diagnostic purposes.

An elevated serum anti-thyroid peroxidase (TPOAb) is widely considered the best serological marker of Hashimoto's thyroiditis, and it is detectable in about 95\% of patients with clinical Hashimoto's thyroiditis as defined by the spontaneous onset of hypothyroidism when neither surgery nor radioiodine ablation has been performed (5). However, young patients often have lower titers of TPOAb, and even occasional negative levels, despite clinically proven disease. Thyroid sonography characteristically shows a diffusely heterogeneous parenchyma, reduced echogenicity, or multiple illdefined small nodules (pseudo-nodules) in the setting of clinical Hashimoto's thyroiditis. Still, data suggest that ultrasound patterns suggestive of Hashimoto's thyroiditis may also be found in about $20 \%$ of individuals with negative TPOAb concentration (6). Of note, in many countries, thyroid ultrasound is only performed if clinical indications beyond hypothyroidism are present, for instance, palpable goiter or thyroid mass. These data suggest concordance of serology and sonography with gold standard histopathology may be imperfect.

The prevalence of elevated thyroid antibodies (TPOAb and anti-thyroglobulin (TgAb)) in the general population is $\sim 10 \%$, but it increases with age and is more common in women $(7,8)$. However, the prevalence of spontaneous hypothyroidism (clinical Hashimoto's thyroiditis) in iodine-sufficient populations is much lower ( 1-2\%) (9). As the clinical onset of hypothyroidism can be subtle and difficult to predict, it is not surprising that the incidence of Hashimoto's thyroiditis based on serological criteria is much higher than that diagnosed by biochemical hypothyroidism. Similarly, it is well known that most individuals with elevated (especially mildly elevated) TPOAb may never evolve into clinical disease (8).

The Japanese Guidelines for the Diagnosis of Chronic Thyroiditis (10) include both clinical (diffuse swelling of the thyroid gland not due to other conditions) and laboratory criteria (positive thyroid auto-antibodies or lymphocytic infiltration of the gland) necessary to confirm Hashimoto's thyroiditis. According to these guidelines, the presence of a diffuse heterogeneous thyroid parenchyma on ultrasound is only supportive of Hashimoto's thyroiditis.
To date, no study has evaluated the concordance of both serologic and sonographic markers with the gold-standard histopathologic finding of Hashimoto's thyroiditis. Such an investigation would be highly difficult to do in most populations because thyroidectomy is rarely a treatment for this disorder. However, a population of patients undergoing evaluation of clinically relevant thyroid nodules affords a unique opportunity to investigate this question in a population in whom surgery was otherwise indicated. Thus, employing this approach, we sought to determine concordance of the different diagnostic tools for Hashimoto's thyroiditis in a cohort of patients undergoing thyroidectomy.

\section{Methods}

We performed a retrospective cohort analysis among adult ( $\geq 18$-year-old) patients consecutively evaluated between 1995 and 2017 at the Brigham and Women's Hospital (BWH) for a thyroid nodule leading to surgical thyroidectomy. During this time, a total of 3158 patients ultimately underwent thyroidectomy following nodule evaluation. Given the decreased accuracy of ultrasound in assessing thyroid parenchyma in the presence of extensive multinodularity, patients with six or more nodules (each $>1 \mathrm{~cm})(n=66)$ were excluded (11). The final cohort for analysis only includes patients in whom pre-surgical information inclusive of both ultrasound and TPOAb concentrations were available $(n=825)$. Characteristics of these patients are shown in Table 1. Notable in this cohort, most patients were biochemically euthyroid (normal serum thyrotropin) as guidelines current to the time recommended assessment of serum thyrotropin prior to nodule evaluation, and most patients in this cohort did not have clinical evidence of hypothyroidism.

Clinical, laboratory, sonographic and pathologic information was collected from review of the medical record for all patients. Ultrasound was performed using a $10-22 \mathrm{mHz}$ transducer and read by one of five radiologists with expertise in thyroid sonography. The terms 'diffusely heterogeneous parenchyma' and 'consistent with Hashimoto's disease (or Hashimoto's thyroiditis)' were considered positive for Hashimoto's thyroiditis. In rare cases where sonographic interpretation was unclear, a second analysis of the images by a blinded expert was performed. Thyroid echogenicity was determined by comparing echoes from the thyroid to the surrounding neck muscles. Focal areas of reduced echogenicity or heterogeneity of the thyroid were not considered diagnostic of Hashimoto's thyroiditis. 
Table 1 Characteristics of patients included in the analysis cohort.

\begin{tabular}{l} 
Characteristic \\
\hline Number \\
Age, years (mean \pm S.D.) \\
Elevated TPOAb $(n(\%))$ \\
Diffuse sonographic heterogeneity $(n(\%))$ \\
Histopathological Hashimoto's thyroiditis $(n(\%))$ \\
\hline
\end{tabular}

\begin{tabular}{c}
\hline Male \\
\hline 76 \\
$52.7 \pm 14.8$ \\
$14(18.4 \%)$ \\
$15(19.7 \%)$ \\
$14(18.4 \%)$ \\
\hline
\end{tabular}

\begin{tabular}{c}
\hline Female \\
\hline 749 \\
$47.0 \pm 12.9$ \\
$221(29.5 \%)$ \\
$182(24.3 \%)$ \\
$263(35.1 \%)$ \\
\hline
\end{tabular}

\begin{tabular}{c}
\hline Total \\
\hline 825 \\
$47.5 \pm 13.1$ \\
$235(28.5 \%)$ \\
$197(23.9 \%)$ \\
$277(33.6 \%)$ \\
\hline
\end{tabular}

We recorded laboratory data performed closest to the date of thyroid ultrasound and prior to thyroidectomy. One of four different assays were used for measurement of TPOAb over 20 years. Before 2013, TPOAb was detected by chemiluminescent assays (Advia ${ }^{\circledR}$ Centaur $^{\mathrm{TM}}$, Bayer; Advia ${ }^{\circledR}$ Centaur XP (CEN), Siemens Healthcare Diagnostics; ELISA TPO kit, Alpco) with TPOAb titers greater than $20 \mathrm{IU} / \mathrm{mL}$ considered abnormal. From 2013 to 2017, serum concentration of TPOAb was determined by chemiluminescence method (Cobas ${ }^{\circledR}$ e 601 analyzer, Roche Diagnostics) with a normal reference range of 0.0 $33.9 \mathrm{IU} / \mathrm{mL}$.

As this cohort reflected a population undergoing evaluation of clinically relevant thyroid nodules, patients were referred for thyroid surgery in the majority of cases due to abnormal cytologic findings concerning for malignancy. Other surgical indications included symptomatic goiter and cosmetic reasons. Following surgery, a histological diagnosis of Hashimoto's thyroiditis was based on the presence of a diffuse infiltration of the gland by lymphocytes, with oncocytic change and the formation of germinal centers, associated with fibrosis or atrophy. The nodule histological diagnoses proved to be benign $(n=367)$, papillary thyroid cancer $(n=420)$, follicular thyroid cancer $(n=24)$, medullary thyroid cancer $(n=3)$, poorly differentiated/anaplastic thyroid cancer $(n=7)$ and other non-thyroid malignancy $(n=4)$. Pathologists were blinded with the information whether or not the patient had serological and sonographic markers for Hashimoto's thyroiditis.

Summary data are presented as mean with standard deviation (S.D.) or median-interquartile ranges (IQR) for continuous values or as absolute numbers-percentages for categorical variables. The $t$-test, Mann-Whitney $U$ test, chisquared $\left(\chi^{2}\right)$ or Fisher's exact were used to compare means, medians or proportions of variables between groups. Concordance between different tests for Hashimoto's thyroiditis diagnosis was assessed using Cohen's kappa coefficient $(k)$. The interpretation of $k$ values were the following: $k \leq 0.20$ (poor agreement); $k=0.21-0.40$ (fair agreement); $k=0.41-0.60$ (moderate agreement); $k=0.61-$ 0.80 (good agreement) and $k=0.81-1.00$ (very good agreement) (12). Diagnostic sensitivity (true positive / (true positive + false negative)), specificity (true negative/(true negative + false positive)), positive predictive value (true positive / (true positive + false positive)), negative predictive value (true negative/(true negative + false negative)) and accuracy ((true positive + true negative)/total) of TPOAb and ultrasound for Hashimoto's thyroiditis was calculated on the basis of histopathological results for the entire cohort. To explore the effect of different TPOAb cutoffs for reflex Hashimoto's thyroiditis on histopathology, we performed receiver-operating characteristic (ROC) curve analysis. The sensitivity and specificity of TPOAb for detecting Hashimoto's thyroiditis on histopathology were established for values across the TPOAb range. A two-sided $P$ value $<0.05$ was considered significant. All calculations were performed using IBM SPSS Statistics Software (IBM), version 25 .

This study was conducted in compliance with a protocol that was approved by the Brigham and Women's Hospital Institutional Review Board, allowing analysis of de-identified information from retrospective cases to be collective for combined analyses in a blinded fashion. Data were reported according to the Standards for Reporting Diagnostic Accuracy (STARD) 2015 (13).

\section{Results}

A total of 825 patients were included in the final analysis for this study, all of whom had thyroid ultrasound and measurement of TPOAb performed, and also underwent thyroidectomy allowing histopathologic analysis. The mean age of participants was $47.5 \pm 13.1$ years, and the majority were female (90.8\%). Among the entire cohort, serum TPOAb was elevated in 235 (28.5\%), while sonographic patterns of diffuse heterogeneity were described in 197 (23.9\%). Histopathology confirmed Hashimoto's thyroiditis in 277 (33.6\%) patients. However, only 102 of 825 (12.4\%) patients (or 102 of 396 patients with at least one positive marker of Hashimoto's thyroiditis) proved positive for all three diagnostic variables simultaneously (serological, sonographic, and 


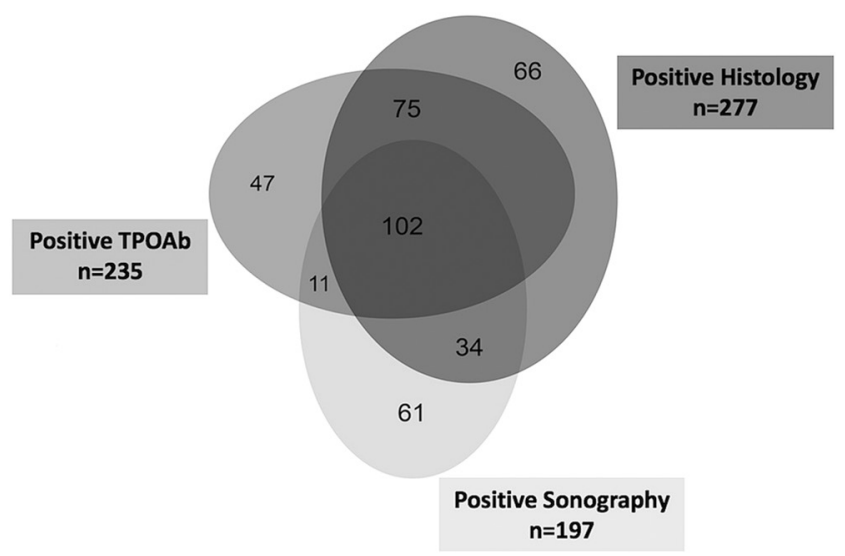

Figure 1

Overlap of positive serologic, sonographic, and histopathologic findings for Hashimoto's thyroiditis, among 825 patients. Included below are the 396 patients with a positive finding in at least one test.

histopathological). The lack of concordance for these three variables is shown in Figure 1.

Using either TPOAb or histopathology as diagnostic criteria, the prevalence of Hashimoto's thyroiditis was higher in females than in males (TPOAb: 29.5 vs $18.4 \%$, $P=0.004$; histopathology: 35.1 vs $18.4 \%, P=0.003)$. Using histopathology as the gold standard for diagnosis of Hashimoto's thyroiditis, the sensitivity, specificity, positive predictive value (PPV), negative predictive value (NPV), and accuracy of an elevated TPOAb for detection of disease was 63.9, 89.4, 75.3, 83.1 and 53.3\%, respectively. The finding of diffuse heterogeneity on ultrasound proved inferior compared to TPOAb as the sensitivity, specificity, PPV, NPV and accuracy of ultrasound were 49.1, 88.9, 69, 77.6 and $38 \%$, respectively (Table 2 ).

We applied calculation of the kappa coefficient $(k)$ to examine the diagnostic agreement between TPOAb measurement, thyroid ultrasound, and histopathology in regard to diagnosis of Hashimoto's thyroiditis. The presence of an elevated serum TPOAb showed the highest diagnostic agreement with histopathologic evidence of Hashimoto's thyroiditis $(k=0.55,95 \%$ confidence interval (CI): $0.493-$ $0.615, P<0.001)$, while ultrasound was less concordant ( $k=0.40,95 \%$ CI: 0.342-0.476, $P<0.001$; Table 3).

Realizing there is a spectrum of TPOAb elevation beyond the reference range (two times, three times or four times, and so forth beyond the upper limit of reference range for each assay), we performed ROC analysis to identify the best TPOAb concentration cutoff predicting the histopathologic presence of Hashimoto's thyroiditis on histopathology. The optimal elevation was 2.11-fold the upper limit of normal TPOAb as shown in Figure 2.

\section{Discussion}

The histopathologic diagnosis of Hashimoto's thyroiditis is made following the microscopic identification of chronic lymphocytic thyroiditis. However, as most patients do not undergo surgical resection, the clinical diagnosis has long been made through detection of elevated serum TPOAb or presence of diffuse sonographic heterogeneity. In a population of euthyroid individuals presenting for nodule evaluation, these data demonstrate that such clinical and histopathologic variables are discordant. Of 396 patients found to have one or more variables positive for Hashimoto's thyroiditis, only 102 (26\%) were positive for all three. The agreement of these variables is best considered moderate, with kappa coefficients generally ranging from 0.4 to 0.6. However, with higher TPOAb concentration, concordance with histopathologic truth increases. Together, these data suggest a cautionary approach to their use in a euthyroid, general population without clinical evidence of disease or thyroid dysfunction.

Two findings in this study cohort are notable and may contribute to a broader understanding of Hashimoto's thyroiditis itself. The first is that our study population was generally euthyroid as defined by a normal thyrotropin. This finding is critical because much of the available

Table 2 Diagnostic performance of detecting an elevated TPOAb concentration, or diffuse sonographic heterogeneity, for the diagnosis of Hashimoto's thyroiditis.

\begin{tabular}{|c|c|c|c|c|c|}
\hline & Sensitivity & Specificity & PPV & NPV & Accuracy \\
\hline Elevated TPOAb & $63.9 \%$ (57.9-69.6) & $89.4 \%$ (86.5-91.9) & $75.3 \%(69.3-80.7)$ & $83.1 \%(79.8-86.0)$ & $53.3 \%(44.4-61.5)$ \\
\hline $\begin{array}{l}\text { Diffuse sonographic } \\
\text { heterogeneity }\end{array}$ & $49.1 \%(43.1-55.2)$ & $88.9 \%(85.9-91.4)$ & $69.0 \%(62.0-75.4)$ & $77.6 \%(74.1-80.8)$ & $38.0 \%(29.0-46.6)$ \\
\hline $\begin{array}{l}\text { NPV, negative predictive } \\
\text { Sensitivity = true positive } \\
\text { positive / (true positive + } \\
\text { negative) / total. }\end{array}$ & $\begin{array}{l}\text { V, positive predictive } \\
\text { ositive + false negativ } \\
\text { itive); negative predi }\end{array}$ & e value = true negati & $\begin{array}{l}\text { / (true negative }+\mathrm{fa} \\
\text { true negative }+ \text { false }\end{array}$ & $\begin{array}{l}\text { ultrasound. } \\
\text { ositive); positive predi } \\
\text { ative); accuracy = (true }\end{array}$ & $\begin{array}{l}\text { ve value }=\text { true } \\
\text { sitive + true }\end{array}$ \\
\hline
\end{tabular}


Table 3 Agreement between various diagnostic tests predicting Hashimoto's thyroiditis.

\begin{tabular}{|c|c|c|}
\hline & Kappa (к) & $95 \% \mathrm{Cl}$ \\
\hline TPOAb vs ultrasound & 0.356 & $0.285-0.427$ \\
\hline TPOAb vs histopathology & 0.554 & $0.493-0.615$ \\
\hline $\begin{array}{l}\text { Ultrasound vs } \\
\text { histopathology }\end{array}$ & 0.409 & $0.342-0.476$ \\
\hline
\end{tabular}

TPOAb, anti-thyroid peroxidase antibody; $\mathrm{Cl}$, confidence interval.

literature on diagnostic studies in the setting of Hashimoto's thyroiditis has been performed on hypothyroid patients or those already receiving levothyroxine replacement therapy. When the disease has progressed to a point of tissue destruction requiring hormonal replacement, it is logical to think the biochemical and sonographic variables would be much more likely to be abnormal and concordant. Second, the prevalence of Hashimoto's thyroiditis in this study was higher than often reported in the current literature even when euthyroid patients are analyzed. Epidemiological studies report a prevalence of elevated TPOAb and TgAb in approximately $10-27 \%$ of healthy populations $(7,8,9)$. In our cohort, the prevalence of positive TPOAb was $28.5 \%$. This may relate to differences between the populations used for studies, especially acknowledging that all patients

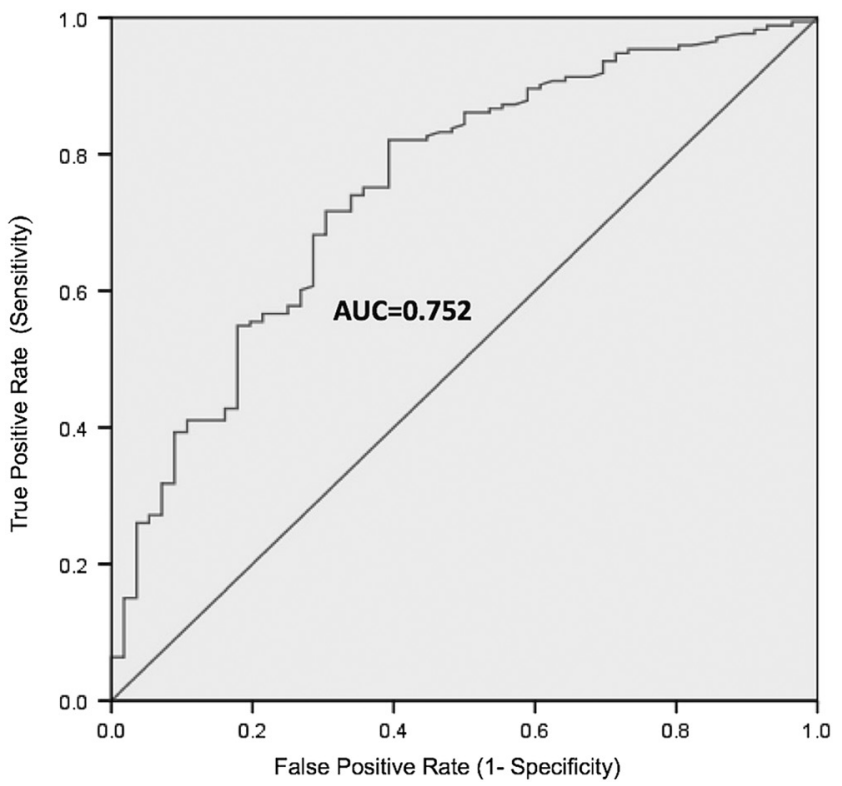

\section{Figure 2}

ROC curve analysis depicting the optimal elevated TPOAb titer for predicting the presence of Hashimoto's thyroiditis on histopathology. The optimal cutoff was a 2.11-fold increase above the upper reference range value (sensitivity: 0.82 ; specificity: 0.61). in our analysis were being evaluated for suspected thyroid nodules. Such referral is based upon suspicion that a nodule was felt or detected on exam or other imaging. As many nodules can cause similar tissue fullness or goiter on examination comparable to that noted in the setting of Hashimoto's thyroiditis, it is not surprising that this higher prevalence was identified (9).

Notable to our data, both the presence of an elevated TPOAb or diffuse heterogeneity on ultrasound examination showed high specificity (89.4 and 88.9\%, respectively) and reasonable sensitivity (63.9 and 49.1\%, respectively) in identifying Hashimoto's thyroiditis. These data suggest that a normal TPOAb or normal thyroid parenchyma on ultrasound are both of considerable value for excluding Hashimoto's thyroiditis as negative predictive values were approaching $80 \%$ or higher. However, positive predictive value of elevated TPOAb or diffuse heterogeneity was lower, though still noting that nearly three of every four with a positive finding did prove to have Hashimoto's thyroiditis. Together, these findings support the continued use of these variables as they do contribute clinical information though are far from perfect in terms of positively or negatively predicting disease.

Similar to our study, Rho et al. (14) studied 598 patients who also underwent thyroid nodule surgery, finding a sensitivity of elevated TPOAb to predict pathologic Hashimoto's thyroiditis at 48.6\%, while specificity was $96.9 \%$. However, after excluding patients with non-Hashimoto type of lymphocytic thyroiditis, TPOAb sensitivity was much higher (57.7\%). Chehade et al. (15) previously investigated the concordance rate between cytological diagnosis of Hashimoto's thyroiditis and positive TPOAb in people with nodular goiter. The sensitivity and specificity of TPOAb in detecting Hashimoto's thyroiditis on cytology were 76.4 and $94.2 \%$, respectively. These data lend support for our findings.

Though the current study is powered by large number of patients in whom post-surgical histopathology assessment was followed by evaluations on serological and sonographic markers prior to surgery, we acknowledge the limitations to our study. First, we could not access all TSH levels prior to the time of ultrasound and surgery or the use of levothyroxine replacement therapy on all patients. Thus, while we are confident the majority of our patients were indeed euthyroid as guideline protocols were followed, we were unable to assess specific TSH concentration in our cohort. Perhaps some patients with TSH in the upper portion of the reference range may have proved to have better concordance of tests compared to those with TSH values $<2.0 \mathrm{mIU} / \mathrm{L}$. Second, 
we recognize that ultrasound is an operator-dependent examination with subjective interpretation and that findings of diffuse heterogeneity may vary by user or with different machines or transducers. Similarly, excessive amplification may mask reduction of echogenicity, while limited amplification may cause an erroneous diagnose of thyroid hypoechogenicity (16). Finally, quite a number of patients who underwent thyroidectomy during the study period had to be removed from the analysis cohort due to lack of pre-surgical TPOAb measurement. This may lead to selection bias, therefore exert influence on the prevalence of Hashimoto's thyroiditis in the population, which has implications of the interpretation of positive and negative predictive value. More studies are needed to validate our findings in the future. Ideally, studies of this kind should consist of a large number of patients whose TSH, TPOAb and ultrasound are examined right before the surgery, and detailed histopathology reports are available as well.

In conclusion, in this large cohort analysis of a mostly euthyroid population presenting for thyroid nodule analysis, elevated TPOAb and diffuse sonographic heterogeneity both demonstrated only moderate concordance with the histopathological diagnosis of Hashimoto's thyroiditis. Of these variables, serum TPOAb was superior, especially when titers were highly elevated. Nonetheless, both variables, when identified, should not be considered diagnostic of Hashimoto's thyroiditis. Rather, they represent supportive evidence favoring Hashimoto's thyroiditis but demonstrate a $25-30 \%$ false positive.

\section{Declaration of interest}

The authors declare that there is no conflict of interest that could be perceived as prejudicing the impartiality of the research reported.

\section{Funding}

This research did not receive any specific grant from any funding agency in the public, commercial or not-for-profit sector.

\section{References}

1 Caturegli P, De Remigis A, Chuang K, Dembele M, Iwama A \& Iwama S. Hashimoto's thyroiditis: celebrating the centennial through the lens of the Johns Hopkins Hospital surgical pathology records. Thyroid 201323 142-150. (https://doi.org/10.1089/thy.2012.0554)

2 Golden SH, Robinson KA, Saldanha I, Anton B \& Ladenson PW. Clinical review: prevalence and incidence of endocrine and metabolic disorders in the United States: a comprehensive review. Journal of Clinical Endocrinology \& Metabolism 200994 1853-1878. (https://doi.org/10.1210/jc.2008-2291)

3 Jacobson DL, Gange SJ, Rose NR \& Graham NM. Epidemiology and estimated population burden of selected autoimmune diseases in the United States. Clinical Immunology \& Immunopathology 199784 223-243. (https://doi.org/10.1006/clin.1997.4412)

4 McLeod DS \& Cooper DS. The incidence and prevalence of thyroid autoimmunity. Endocrine 201242 252-265. (https://doi.org/10.1007/ s12020-012-9703-2)

5 Caturegli P, De Remigis A \& Rose NR. Hashimoto thyroiditis: clinical and diagnostic criteria. Autoimmunity Reviews 201413 391-397. (https://doi.org/10.1016/j.autrev.2014.01.007)

6 Biondi B \& Cooper DS. The clinical significance of subclinical thyroid dysfunction. Endocrine Reviews 200829 76-131. (https://doi. org/10.1210/er.2006-0043)

7 Hollowell JG, Staehling NW, Flanders WD, Hannon WH, Gunter EW, Spencer CA \& Braverman LE. Serum TSH, T(4), and thyroid antibodies in the United States population (1988 to 1994): National Health and Nutrition Examination Survey (NHANES III). Journal of Clinical Endocrinology \& Metabolism 200287 489-499. (https;//doi. org/10.1210/jcem.87.2.8182)

8 Vanderpump MP, Tunbridge WM, French JM, Appleton D, Bates D, Clark F, Grimley Evans J, Hasan DM, Rodgers H \& Tunbridge F. The incidence of thyroid disorders in the community: a twenty-year follow-up of the Whickham Survey. Clinical Endocrinology 199543 55-68. (https://doi.org/10.1111/j.1365-2265.1995.tb01894.x)

9 Vanderpump MP. The epidemiology of thyroid disease. British Medical Bulletin 201199 39-51. (https://doi.org/10.1093/bmb/ldr030)

10 Japan Thyroid Association. Guidelines for the diagnosis of chronic thyroiditis. (available at: http://www.japanthyroid.jp/en/guidelines. html\#Chr; Accessed on 10 June 2019).

11 Patel S, Giampoli E, Oppenheimer D, Montoya S, Rupasov A \& Dogra V. Sonographic features of diffuse Hashimoto thyroiditis: determining sensitivity of features and predictors of malignancy. American Journal of Sonography 20181 1-7. (https://doi.org/10.25259/ AJS-7-2018)

12 Landis JR \& Koch GG. An application of hierarchical kappatype statistics in the assessment of majority agreement among multiple observers. Biometrics 197733 363-374. (https://doi. org/10.2307/2529786)

13 Bossuyt PM, Reitsma JB, Bruns DE, Gatsonis CA, Glasziou PP, Irwig L, Lijmer JG, Moher D, Rennie D, de Vet HC, et al. STARD 2015: an updated list of essential items for reporting diagnostic accuracy studies. BMJ 2015351 h5527. (https://doi.org/10.1136/ bmj.h5527)

14 Rho MH, Kim DW, Hong HP, Park YM, Kwon MJ, Jung SJ, Kim YW $\&$ Kang T. Diagnostic value of antithyroid peroxidase antibody for incidental autoimmune thyroiditis based on histopathologic results. Endocrine 201242 647-652. (https://doi.org/10.1007/s12020-0129695-y)

15 Chehade JM, Lim W, Silverberg AB \& Mooradian AD. The incidence of Hashimoto's disease in nodular goitre: the concordance in serological and cytological findings. International Journal of Clinical Practice 201064 29-33. (https://doi.org/10.1111/j.17421241.2008.01942.x)

16 Pedersen OM, Aardal NP, Larssen TB, Varhaug JE, Myking O \& Vik-Mo H. The value of ultrasonography in predicting autoimmune thyroid disease. Thyroid 200010 251-259. (https://doi.org/10.1089/ thy.2000.10.251) 